\title{
Explaining the Sr and Ba Scatter in Extremely Metal-Poor Stars
}

\author{
W. Aoki \\ National Astronomical Observatory of Japan, 2-21-1 Mitaka, Tokyo, 181-8588, Japan \\ aoki.wako@nao.ac.jp \\ T. Suda \\ National Astronomical Observatory of Japan, 2-21-1 Mitaka, Tokyo, 181-8588, Japan \\ takuma.suda@nao.ac.jp \\ R.N. Boyd \\ National Astronomical Observatory of Japan, 2-21-1 Mitaka, Tokyo, 181-8588, Japan \\ richard11boyde@comcast.net \\ T. Kajino ${ }^{1}$ \\ National Astronomical Observatory of Japan, 2-21-1 Mitaka, Tokyo, 181-8588, Japan \\ kajino@nao.ac.jp \\ and \\ M.A. Famiano ${ }^{2}$ \\ National Astronomical Observatory of Japan, 2-21-1 Mitaka, Tokyo, 181-8588, Japan \\ michael.famiano@wmich.edu
}

Received

accepted

\footnotetext{
${ }^{1}$ Dept. of Astronomy, Graduate School of Science; Univ. of Tokyo, 7-3-1, Hongo, Bunkyoku, Tokyo 113-0033, Japan

${ }^{2}$ Dept. of Physics and Joint Institute for Nuclear Astrophysics, Western Michigan University, 1903 W. Michigan Avenue, Kalamazoo, MI 49008-5252, USA
} 


\begin{abstract}
Compilations of abundances of Strontium and Barium in extremely metalpoor stars show that an apparent cutoff is observed for $[\mathrm{Sr} / \mathrm{Ba}]$ at $[\mathrm{Fe} / \mathrm{H}]<-3.6$ and large fluctuations for $[\mathrm{Fe} / \mathrm{H}]>-3.6$ with a clear upper bound depending on metallicity. We study the factors that place upper limits on the logarithmic ratio $[\mathrm{Sr} / \mathrm{Ba}]$. A model is developed in which the collapses of type II supernovae are found to reproduce many of the features seen in the data. This model is consistent with galactic chemical evolution constraints of light-element enrichment in metalpoor stars. Effects of turbulence in an explosive site have also been simulated, and are found to be important in explaining the large scatter observed in the $[\mathrm{Sr} / \mathrm{Ba}]$ data.
\end{abstract}

Subject headings: stars: Population II — nuclear reactions, nucleosynthesis, abundances — black hole physics — Galaxy: evolution — equation of state 


\section{Introduction}

Nuclei heavier than iron and nickel are mostly made either in the s-process (slow neutron-capture process) or r-process (rapid neutron-capture process) (B2FH 1957; Wallerstein et al. 1997), which is thought to produce half the nuclides heavier than Fe and nearly all actinides observed in most metal-poor stars. Although effects of the s-process can occur in first generation stars (Goriely \& Siess 2001; Suda et al. 2004; Nishimura et al. 2009), the s-process appears not to be responsible for the enrichment of the interstellar medium with the elements produced in the early life of the galaxy because it requires longer timescales to populate the ISM with its products. The r-process, however, produces its nuclei earlier in the galactic history. Because massive stars produce most of the required conditions for the r-process, they have long been supposed to synthesize its nuclides. However, detailed calculations (Fischer et al. 2010; Hudepohl et al. 2010; Goriely et al. 2011) have suggested that massive stars may not fulfill all of the r-process conditions. We present data that sheds important new light on this question.

Stars with masses greater than $8 M_{\odot}$ are thought to become core-collapse supernovae. Those less than $25 M_{\odot}$ will form neutron stars, emitting neutrinos and driving some of their newly synthesized nuclei into the interstellar medium. Those more massive than about $40 M_{\odot}$ are thought to collapse directly to black holes, contributing nothing to the interstellar medium. Stars with $25 \leq M \leq 40 M_{\odot}$ are thought to collapse to neutron stars then, as some of the expelled material falls back onto the neutron star, to black holes (Fryer) 2003; Heger et al. 2003), producing "accretion-induced black holes" and, assuming some material can escape, would be expected to contribute some newly synthesized nuclides to the interstellar medium.

We report on some striking astronomical data from extremely metal-poor (EMP) stars, those having $[\mathrm{Fe} / \mathrm{H}]<-2.5$. Their metals are thought to have been produced in the first 
generation massive stars that were the progenitors of the ones currently observed. Because these stars have very low abundances of anything except primordial hydrogen and helium, their abundances of heavy nuclei are presumed to arise primarily from the r-process. We concentrate on elements made in the r-process and that are easy to detect in these stars: strontium and barium.

Previous models (Qian \& Wasserburg 2008; Wanajo et al. 2011; Travaglio et al. 2004) have described separate r-process sites as the cause of an enrichment of light neutron-capture r-process elements. We contrast our model with the "weak" r-process and the light element primary process (LEPP) by describing an enrichment in the lighter neutron-capture elements (Honda et al. 2006) that is a natural consequence of a single site but with differing levels of enrichment of light and heavier neutron-capture elements. Our model produces the r-process from an explosive event (e.g., a type II supernova), while the r-process ejecta is prohibited in some fraction of those events from escaping due to a subsequent collapse of the proto-neutron star to a black hole. The collapse time in this event relative to the r-process ejection time will determine how much r-process material is ejected, as well as its composition.

\section{Observational Data}

Data were taken from the SAGA Database (Suda et al. 2008). The sample includes data from 186 papers and 4270 stars (1488 unique stars) dealing with observations of metal-poor halo stars published prior to 2012. The number of stars for which $[\mathrm{Fe} / \mathrm{H}]<-2.5$ is 428, reducing to 361 stars if carbon-enhanced EMP stars are excluded. This sample could suffer errors due to systematic offsets between different studies; a comparison of results for the same stars but from different studies shows that these can sometimes be as large as a factor of 3. We thus selected the results of $\mathrm{Sr}$ and $\mathrm{Ba}$ from observations in 
which both elements are reported in the same paper - 260 total stars. The abundances in this database were all obtained assuming local thermodynamic equilibrium (LTE). The scatter found in $\mathrm{Sr} / \mathrm{Ba}$ as well as in $\mathrm{Sr} / \mathrm{Fe}$ and $\mathrm{Ba} / \mathrm{Fe}$ in that sample in the metallicity range of $-3.6<[\mathrm{Fe} / \mathrm{H}]<-3.0$ is observed to be a factor of 100 or more, much larger than the observational errors.

Figure 1 shows the astronomical data for $[\mathrm{Sr} / \mathrm{Fe}],[\mathrm{Ba} / \mathrm{Fe}]$, and $[\mathrm{Sr} / \mathrm{Ba}]$ from the data set described above, showing a wide range of $[\mathrm{Sr} / \mathrm{Ba}],[\mathrm{Sr} / \mathrm{Fe}]$, and $[\mathrm{Ba} / \mathrm{Fe}]$ values at each value of $[\mathrm{Fe} / \mathrm{H}]$. Our interpretation of these features will be given in subsequent sections; the curves in Figure 1 that overlay the data, reflect these efforts.

We find three remarkable features in the $[\mathrm{Sr} / \mathrm{Ba}]$ distribution.

1. Almost all stars appear in the range of the functional $[\mathrm{Sr} / \mathrm{Ba}]<-1.5-[\mathrm{Fe} / \mathrm{H}]$. There are two exceptions in $[\mathrm{Fe} / \mathrm{H}]<-2.0$. One of them is $\mathrm{BD}+80^{\circ} 245([\mathrm{Fe} / \mathrm{H}]=-2.09$, $[\mathrm{Sr} / \mathrm{Ba}]=+1.04)$, which is a well known " $\alpha$-deficient" star (e.g., $[\mathrm{Mg} / \mathrm{Fe}]=-0.22$ ) having exceptionally low abundances of neutron-capture elements ([Sr/Fe $]=-0.85$ and $[\mathrm{Ba} / \mathrm{Fe}]=-1.89$ which was studied by Ivans et al. (2003)). The other is HE0029-1839 studied by Barklem et al. (2005), for which little information exists.

2. There are only a few stars with $[\mathrm{Sr} / \mathrm{Ba}]<-0.5$. We present calculations that show that this range is not expected from the r-process or from tr-process, which we discuss in the next section. The exceptions are as follows:

- CS 30322-023 ([Fe/H] =-3.25, $[\mathrm{Sr} / \mathrm{Ba}]=-1.10)$ : a moderately carbon-enhanced star $([\mathrm{C} / \mathrm{Fe}]=+0.6)$ with enhancement of nitrogen and Ba (Masseron et al. 2006; Aoki et al. 2007).

- CS 29493-090 ([Fe/H] =-2.82, $[\mathrm{Sr} / \mathrm{Ba}]=-1.41)$ : a moderately carbon-enhanced star $([\mathrm{C} / \mathrm{Fe}]=+0.74)$ with excess of Ba (Barklem et al. 2005). 
- CS 22946-011 ([Fe/H]=-2.59, [Sr/Ba $]=-0.99)$ and CS 22941-005 $([\mathrm{Fe} / \mathrm{H}]=-2.43$, $[\mathrm{Sr} / \mathrm{Ba}]=-0.77)$ : binaries with excesses of $\mathrm{Ba}([\mathrm{Ba} / \mathrm{Fe}]=+1.26$ and +0.34 , respectively: Preston \& Sneden (2000) ). CS 22950-173 $([\mathrm{Fe} / \mathrm{H}]=-2.5,[\mathrm{Sr} / \mathrm{Ba}]=-$ 0.72) which also belongs to be a binary system, though $\mathrm{Ba}$ is not enhanced $([\mathrm{Ba} / \mathrm{Fe}]=-0.04:$ Preston \& Sneden $(2000))$. The $\mathrm{CH}$ feature would not be detected due to high temperature $(\sim 6800 \mathrm{~K})$.

- $\operatorname{HE} 0305-4520([\mathrm{Fe} / \mathrm{H}]=-2.91,[\mathrm{Sr} / \mathrm{Ba}]=-1.25)$ : Ba is slightly enhanced $([\mathrm{Ba} / \mathrm{Fe}]=+0.59)$, while an excess of carbon is not clear $([\mathrm{C} / \mathrm{Fe}]=+0.33$ : Barklem et al. (2005)).

Hence, except for HE0305-4520, all the above stars are (candidate) carbon-enhanced stars, which can be affected by an s-process contribution through mass transfer in binary systems. HE0305-4520 could also be slightly affected by s-process. In the objects with low $\mathrm{Sr}$ abundances, $\mathrm{Sr} / \mathrm{Ba}$ could be driven to very low values even by a small contamination of Ba from the s-process.

There is no object for which s-process contamination is not suspected in the region $[\mathrm{Sr} / \mathrm{Ba}]<-0.5$ given the typical errors $(\sim 0.2$ dex or larger $)$ in $[\mathrm{Sr} / \mathrm{Ba}]$.

3. A cutoff in $[\mathrm{Sr} / \mathrm{Ba}]$ data at $[\mathrm{Fe} / \mathrm{H}] \sim-3.6$, which will be addressed below, is found. There are only four stars (CS 22172-002, CD-38²45, CS 22885-296, and CS 30336-049) in $[\mathrm{Fe} / \mathrm{H}]<-3.6$ for which both of the $\mathrm{Sr}$ and $\mathrm{Ba}$ abundances are determined, and all of them have low $[\mathrm{Sr} / \mathrm{Ba}](<0.05)$. There are roughly three times as many stars with $[\mathrm{Sr} / \mathrm{Ba}]>0.05$ than with $[\mathrm{Sr} / \mathrm{Ba}]<0.05$ in the region $-3.6<[\mathrm{Fe} / \mathrm{H}]<-3.0$. If the same distribution is assumed for $-4.0<[\mathrm{Fe} / \mathrm{H}]<-3.6$, one would expect 12 - 15 stars with $[\mathrm{Sr} / \mathrm{Ba}]>0.05$, instead of the zero stars that are observed, from the four stars with $[\mathrm{Sr} / \mathrm{Ba}]>0.05$.

To confirm this cutoff, we inspected the data with $[\mathrm{Fe} / \mathrm{H}]<-3.6$ in more detail. We 
found 18 objects in this range in our sample, but both $\mathrm{Sr}$ and $\mathrm{Ba}$ abundances were determined only for seven objects, among which three are carbon-enhanced objects. Among the remaining 11 objects for which $[\mathrm{Fe} / \mathrm{H}]<-3.6$, five stars are carbonenhanced, and four have very low $[\mathrm{Sr} / \mathrm{Fe}]$ or low upper limits, suggesting that $\mathrm{Sr} / \mathrm{Ba}$ ratios are also quite low. There is no signature to have high $\mathrm{Sr} / \mathrm{Ba}$ for other two objects although the upper limits for Ba abundances are still weak.

We note that no new stars with both $\mathrm{Sr}$ and Ba measurements for $[\mathrm{Fe} / \mathrm{H}]<-3.6$ were reported by the recent paper to study chemical composition of most metal-poor stars by Yong et al. (2012).

\section{Galactic Chemical Evolution Interpretation and Turbulence}

The most daunting challenges for any attempt to explain the data distribution for the EMP stars are reproduction of the sharp cutoff in $[\mathrm{Sr} / \mathrm{Ba}]$ at $[\mathrm{Fe} / \mathrm{H}]=-3.6$, the explanation of the upper and lower limits as a function of metallicity in that distribution, and the wide dispersion in $[\mathrm{Sr} / \mathrm{Ba}]$.

A reduction in the $\mathrm{Sr}$ and $\mathrm{Ba}$ production in a SN collapse scenario was studied previously (Boyd et al. 2012), using the formalism and results from Woosley et al. (1994). There, the r-process occurs in upper-most 15 shells that appear just at the surface of the nascent neutron star. The mass, thermodynamic trajectories, and initial composition of each

shell are described in Woosley et al. (1994). Those shells were examined in the framework of the ejection time, the black-hole collapse time, and the resultant nucleosynthesis, assuming that the ejection is halted by the collapse to a black hole. We assume that any collapse time occurring prior to the shell ejection time in this model will prevent that shell from being ejected. At present, we extend that model to study the relationship between the stellar 
metallicity, the progenitor mass, the collapse time, and elemental yields. This model is coupled to a galactic chemical evolution (GCE) code (Timmes, Woosley, \& Weaver 1995). The net result is that a more massive progenitor will generally collapse earlier, resulting in ejecta enriched in the lighter neutron-capture elements $(\mathrm{A} \lesssim 130)$ but a lower yield of the heavier elements. This GCE formulation will be described in detail in a subsequent paper (Famiano et al. 2012). Here, we present the general principles.

Previous results have indicated that a truncation in the r-process in type II supernovae due to a collapse of the proto-neutron star to a black hole may be responsible for a relative enrichment of light neutron-capture elements in some metal-poor halo stars produced by a single-site "truncated r-process" (Boyd et al. 2012) (where single-site is defined to be a single episode of star formation after one r-process event). This "tr-process" could be caused by either a dynamic collapse of material below the black hole mass cut or by a change in the neutrino luminosity resulting in an increased electron fraction $Y_{e}$ in the later stages of the r-process, which would result in a reduced synthesis of the r-process nuclei with masses in excess of $\mathrm{A} \sim 130$.

We adopt a model based on prior calculations (Boyd et al. 2012) in which the isotopic yields in a tr-process are directly related to the collapse time in an accretion-induced black hole. In order to produce the chemical evolution results shown, we began with the Ba yields of Cescutti et al. (2006) and performed several calculations iteratively to arrive at the yields shown in Figure 2. These assumed yields reproduce the GCE results shown by the red lines in Figure 1, For stars with $10 \mathrm{M}_{\odot}<\mathrm{M}<30 \mathrm{M}_{\odot}$, the yields are roughtly equal to those of Model 1Max in Cescutti et al. (2006) but slightly less than those of model 2Max in the same reference and less than those of Raiteri et al. (1999) by about a factor of four (for 10-11 $\mathrm{M}_{\odot}$ SNIIs). For $\mathrm{M}<10 \mathrm{M}_{\odot}$ yields are estimated from Figure 3 of Cescutti et al. (2006). The Sr yields (Figure 21) were chosen to reproduce the [Sr/Fe] and [Sr/Ba] GCE 
results in Figure 1. The only significant difference between the Ba yields in this paper and those of Cescutti et al. (2006) corresponds to $30 \mathrm{M}_{\odot}$ stars, which are roughly 30 times higher than those of model 1Max. This produces Ba early in the GCE model, though the contribution is insignificant at late times.

A power law IMF was used with a Salpeter exponent of -1.31. The black hole collapse time was computed based on progenitor mass and metallicity and the nuclear equation of state (EOS) (O'Connor \& Ott 2010). This time was then assumed to be the truncation time in a dynamic tr-process. For later truncation times, mass shells closer to the proto-neutron star core, blown off later in the explosion model, are assumed to be ejected either by achieving a velocity in excess of the escape velocity or perhaps by being ejected in a jet. Later collapse times correspond to larger production yields of heavier r-process elements.

In the r-process, most of the $\mathrm{Sr}$ is produced in the early-stage ejectt in mass shells ejected within the first four seconds post-bounce, while most of the $\mathrm{Ba}$ is produced in the later-stage ejecta of a SNII in mass shells ejected from between four and 18 seconds post-bounce. Thus, the Sr yields in a tr-process are fairly constant with collapse time and fairly close to their complete r-process values except for very short collapse times for which no r-process material is ejected. As only fairly late collapse times will allow for a significant amount of $\mathrm{Ba}$ ejection in the tr-process, $[\mathrm{Sr} / \mathrm{Ba}]$ approaches zero in the later-stage ejecta.

Results from the spherical collapse code GR1D(O'Connor \& Ott 2010) were used to study the relationship between the progenitor star's mass and metallicity and the Sr and $\mathrm{Ba}$ yields in massive stars. It is noted that these yields are minimum yields, since in the non-rotating spherical models employed here, the collapse times are minimum collapse times. Longer collapse times may result from effects such as asymmetric explosions, rotations, and neutrino heating induced by these effects. However, since Sr is produced in 
shells that are ejected very early on, increasing the collapse time will have little effect on the Sr production, while significantly increasing the Ba production.

Ejection of supernova materials into the interstellar medium resulting from turbulence in the stellar interior may also affect the observed abundances. Although it is difficult to simulate such effects in a one-dimensional model, we have performed a simplified analysis that at least suggests the sort of effects that might be observed, and probably to constrain the magnitude of the predictions. This analysis took each of the 15 individual mass shells that were used in our analysis and that could be ejected from the surface of the neutron star. We then assumed that turbulent ejection might allow a single individual shell to be ejected from the star, while the others collapsed onto the proto-neutron star surface. As noted above, the outer shell would be rich in Sr and would have very little Ba, while the inner shells would have much less Sr and would be much richer in Ba. Ejection of only the top or bottom shells should provide limits on the ability of turbulence to explain the range of $[\mathrm{Sr} / \mathrm{Fe}],[\mathrm{Ba} / \mathrm{Fe}]$, and $[\mathrm{Sr} / \mathrm{Ba}]$ observed and might also explain the dispersion in the data that mixing might produce. The $[\mathrm{Sr} / \mathrm{Ba}]$ results from sites ejecting only these shells are shown in the GCE model in Figure 1d.

\section{Results}

Assuming a tr-process model resulting in a reduced $\mathrm{Sr}$ and Ba production, the single-site $[\mathrm{Sr} / \mathrm{Ba}]$ values as a function of metallicity $[\mathrm{Fe} / \mathrm{H}]$ are shown in Figure 1 with a collapse model utilizing the LS220 EOS (Lattimer \& Swesty 2004), which is one of the most widely used EOSs in numerical simulations of supernova explosions. Several other EOSs have been examined, and it has been found that a softer EOS results in progressively larger $[\mathrm{Sr} / \mathrm{Ba}]$ values at lower metallicity (corresponding to earlier times in the galactic evolution produced by stars with $\left.\mathrm{M} \geq 20 \mathrm{M}_{\odot}\right)($ Famiano et al. 2012). 
In Figure 1, two GCE results are presented. One is the metallicity relationship from a model based on the input yields from Figure 2 showing [Sr/Fe], [Ba/Fe], and [Sr/Ba] ratios. It can be seen that those fall within the extremes of the observational data points. The other result is a metallicity relationship corresponding to a GCE model in which all stars with $\mathrm{M} \geq 20 \mathrm{M}_{\odot}$ ultimately collapse to black holes.

In this model, an earlier collapse means that shells closer to the neutron star surface (which produce more Ba) will not be ejected, and the overall Ba ejection (summed over all shells) is reduced. Since Sr is mostly produced in outer shells, it is not reduced as much. The net result is that the calculated lines in the figure will correspond to the minimum in the $[\mathrm{Sr} / \mathrm{Fe}]$ and $[\mathrm{Ba} / \mathrm{Fe}]$ values and a maximum in the $[\mathrm{Sr} / \mathrm{Ba}]$ values. Collapse times range from about $0.25 \mathrm{~s}$ for $40 \mathrm{M}_{\odot}$ stars with a soft EOS to over $3.5 \mathrm{~s}$ for $20 \mathrm{M}_{\odot}$ stars with a stiff EOS.

The results of these calculations can be compared to the astronomical data of [Sr/Ba] discussed here. These observations show a sharp maximum in the $[\mathrm{Sr} / \mathrm{Ba}]$ value for a given metallicity. This could be explained by the tr-process limiting the Sr or Ba production as a function of stellar age or mass. We also note a similarly sharp lower limit in the [Ba/Fe] values at $[\mathrm{Ba} / \mathrm{Fe}]<0$ corresponding to the sharp $[\mathrm{Sr} / \mathrm{Ba}]$ upper limit. However, the lower limit in $[\mathrm{Sr} / \mathrm{Fe}]$ is less distinct. The curves shown in Figure $1 \mathrm{~d}$ represent attempts to study the effects of turbulence by assuming that individual mass shells were ejected into the ISM. It can be seen that these results do span the entire range of observed [Sr/Ba] values, and even exceed the limits of the data. Obviously, in reality one would expect some mixing of shells to occur. As noted above, what is happening qualitatively is that the outermost shell is high in $\mathrm{Sr}$ and low in $\mathrm{Ba}$, so produces a very large $[\mathrm{Sr} / \mathrm{Ba}]$ value, whereas the innermost shells are low in $\mathrm{Sr}$ and high in $\mathrm{Ba}$, so produce the lowest [Sr/Ba] values in the present GCE model. Additional smearing of these results might be expected from other effects that 
are not included in our simple calculations, but the turbulence alone does appear to provide an explanation of the wide scatter observed in the data.

If the atmospheres of these EMP stars are enriched by the tr-process, then the EOS dependence of the collapse time has a direct effect on the $\mathrm{Sr}$ and $\mathrm{Ba}$ production. Since the plotted values of $[\mathrm{Sr} / \mathrm{Ba}]$ are already the maximum values, it would appear that in this model a very stiff EOS cannot produce the large observed values of [Sr/Ba]; a softer EOS is necessary. It's also noted that the upper limit of observed [Sr/Ba] values provides an observationally imposed lower limit on the softness of the EOS.

\section{Conclusions}

Our detailed inspection of Sr and Ba abundances in Galactic metal-poor stars indicates a clear cutoff in the distribution of $[\mathrm{Sr} / \mathrm{Ba}]$ at $[\mathrm{Fe} / \mathrm{H}] \sim-3.6$ and an upper bound as a function of $[\mathrm{Fe} / \mathrm{H}]([\mathrm{Sr} / \mathrm{Ba}]<-1.5-[\mathrm{Fe} / \mathrm{H}])$ as well as a lower bound at $[\mathrm{Sr} / \mathrm{Ba}] \sim-0.5$.

This work lends support to the suggestion (Boyd et al. 2012) that the cutoff at $[\mathrm{Fe} / \mathrm{H}]=-3.6$ for $[\mathrm{Sr} / \mathrm{Ba}]>0.0$ data for EMP stars can be explained by the tr-process, that is, by the collapses to black holes over a fairly wide mass range of progenitor stars. Furthermore, the results of our GCE calculations are found to predict the upper bound in $[\mathrm{Sr} / \mathrm{Ba}]$ in these data.

A fascinating aspect of this work is the possibility that observed minima in $[\mathrm{Ba} / \mathrm{Fe}]$, $[\mathrm{Sr} / \mathrm{Fe}]$, and $[\mathrm{Sr} / \mathrm{Ba}]$ may be directly related to the stiffness of the nuclear EOS. The lower limit of $[\mathrm{Ba} / \mathrm{Fe}]$ as a function of metallicity predicted in our model can be reproduced in a GCE model assuming a contribution to r-process elements from black holes produced in fallback supernovae. As shown in Figure 1 d, the softer the EOS, the lower the calculated $[\mathrm{Ba} / \mathrm{Fe}]$ as a function of $[\mathrm{Fe} / \mathrm{H}]$. Thus the observed lower limit in $[\mathrm{Ba} / \mathrm{Fe}]$ as a function of 
metallicity appears to constrain the lower limit of the stiffness of the EOS. The tendency towards a soft EOS, however, is consistent with prior experiments and observations (Famiano et al. 2006; Steiner, Lattimer, \& Brown 2010). While astronomical observations of neutron star masses are able to predict a lower limit of the stiffness of the EOS, this model suggests a method of determining the upper limit.

Simulations also suggest that turbulence may explain the wide dispersion seen in these data sets, as the values of the predicted distributions do span more than the entire range of the data, and a more realistic model is necessary.

WA and TK were supported by the JSPS Grants-in-Aid for Scientific Research (23224004 and 20244035) of the Ministry of Education, Culture, Sports, Science and Technology of Japan. RNB and MAF acknowledges support from the National Astronomical Observatory and NSF grant \#PHY-1204486. 


\section{REFERENCES}

Aoki, W. et al. 2007, ApJ 655, 492

Barklem, P.S. et al. 2005, A\&A 439, 129

Boyd, R.N., Famiano, M.A., Meyer, B.S., Motizuki, Y., Kajino, T., \& Roederer, I.U. 2012, ApJ 744, L14

Burbidge, E.M., Burbidge, G.R., Fowler, W.A. \& Hoyle, R. 1957, Rev. Mod. Phys. 29, 547

Cescutti, G., Francois, P., Matteucci, F., Cayrel, R., \& Spite, M. 2006, A\&A, 448, 557

Famiano, M.A., Boyd, R.N., Kajino, T., Aoki, W., \& Suda, T. 2012, ApJ, (in preparation)

Famiano, M.A., Liu, T., Lynch, W.G., Rogers, A.M., Tsang, M.B., Wallace, M.S., Charity, R.J., Komarov, S., Sarantites, D.G., \& Sobotka, L.G. 2006, Phys. Rev. Lett., 97, 052701

Fischer, T., Whitehouse, S.C., Mezzacappa, A., Thielemann, F.-K., \& Liebendorfer, M. 2010, A\&A 517, A80

Fryer, C.L. 2003, Class. Quantum Grav. 20, S73

Goriely, S. \& Siess, A. 2001, A\&A 378, L25

Goriely, S., Chamel, N., Janka, H.-T., \& Pearson, J.M. 2011, A\&A 531, A78

Heger, A., Fryer, C.L., Woosley, S.E., Langer, N. \& Hartmann, D.H. 2003, ApJ 591, 288

Honda, S., Aoki, W., Ishimaru, Y, Wanajo, S., \& Ryan, S.G. 2006, ApJ, 643, 1180

Hudepohl, L., Muller, B., Janka, H.-T., Marek, A., \& Raffelt, G.G. 2010, Phys. Rev. Lett 104,251101 
Ivans, I.I., Sneden, C.S., James, C.R., Preston, G.W., Fulbright, J.P., Höflich, P.A., Carney, B.W., \& Wheeler, J.G. 2003, ApJ, 592, 906

Johnson, J.A., Herwig, F., Beers, T.C., \& Christlieb, N. 2007, ApJ, 658, 1203

Lattimer, J.M. \& Swesty, F.D. 1991, Nuc. Phys. A, 553, 131

Masseron, T., van Eck, S., Famaey, B., Goriely, S., Plez, B., Siess, L., Beers, T.C., Primas, F., \& Jorissen, A. 2006, A\&A, 455, 1059

Nishimura, T., Aikawa, M., Suda, T. \& Fujimoto, M.Y. 2009, PASJ 61, 909

O’Connor, E. \& Ott, C.D.2010, Class. Quant. Grav. 27, 114103

Preston, G.W. \& Sneden, C.S. 2000, AJ, 120, 1014

Preston, G.W., Sneden, C.S., Thompson, I.B., Shectman, S.A., Burley, G.S. 2000, AJ, 120, 1014

Qian, Y.-Z \& Wasserburg, G.J. 2008, ApJ 687, 272

Raiteri, C.M., Villata, M., Gallino, R., Busso, M., \& Cravanzola, A. 1999, ApJ, 518, L91

Steiner, A.W., Lattimer, J.M., Brown, E.F. 2010, ApJ, 722, 33

Suda, T., Aikawa, M., Machida, M.N., \& Fujimoto, M.Y. 2004, ApJ 611, 476

Suda, T., Katsuta, Y., Yamada, S, Suwa, T., Ishizuka, C., Komiya, Y., Sorai, K., Aikawa, M., \& Fujimoto, Y. 2008, PASJ 60, 1159

Timmes, F.X., Woosley, S.E., \& Weaver, T.A. 1995, ApJ 98, 617

Travaglio, C., Gallion, R., Arnone, E., Cowan, J., Jordan, F., \& Sneden, C. 2004, ApJ, 601, 684 
Wallerstein, G. et al. 1997, Rev. Mod. Phys. 69, 995

Wanajo, S., Janka, H.-T., \& Muller, B. 2011,ApJ, 765,L15

Woosley, S.E., Wilson, J.R., Mathews, G.J., Hoffman, R.D., \& Meyer, B.S. 1994, ApJ, 433, 229

Yong, D., Norris, J. E., Bessell, M. S., et al. 2012, ApJ, in press, arXiv:1208.3003 

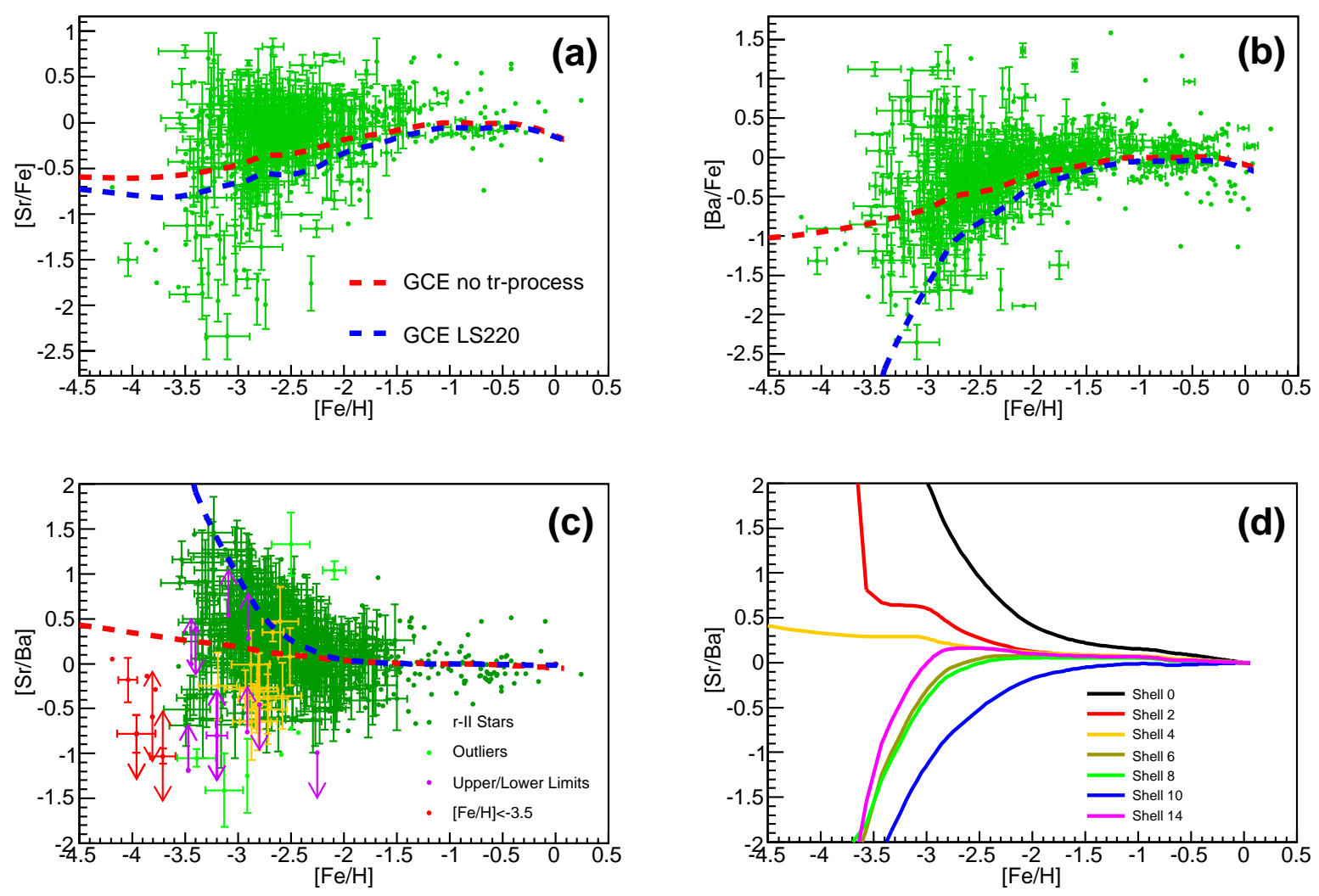

Fig. 1.- Results for (a) [Sr/Fe], (b) [Ba/Fe], and (c) [Sr/Ba] assuming a tr-process. The red dashed line corresponds to a GCE model with no production in a tr-process, and the blue dashed line corresponds to a GCE model with a primary production from a tr-process for all stars with $\mathrm{M} \geq 20 \mathrm{M}_{\odot}$. Plot (d) shows GCE results for single-site tr-process production for turbulent ejection of specific shells assuming only those shells are ejected. 


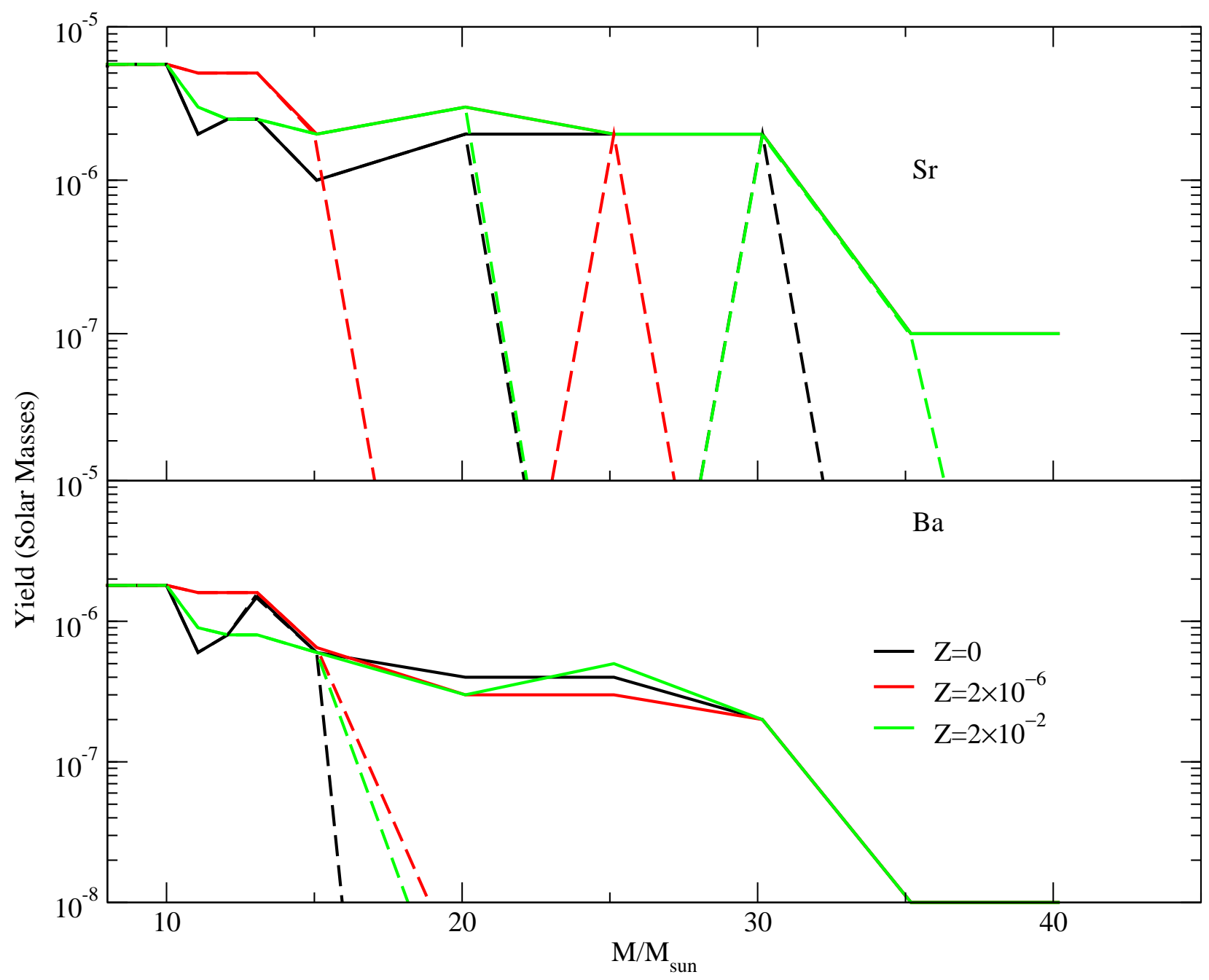

Fig. 2.- Yields of Sr and Ba used for the GCE calculations in this model as a function of stellar mass and metallicity. The solid lines correspond to yields assumed for a primary r-process, while the dotted lines correspond to yields assuming a tr-process resulting from a collapse to a black hole assuming a LS220 EOS. 